\title{
Téoros
}

Revue de recherche en tourisme

\section{Médina de Sousse}

\section{Les enjeux de la gestion touristique d'une ville historique inscrite au patrimoine mondial}

\section{Wided Majdoub}

Volume 30, numéro 2, 2011

Tourisme et patrimoine mondial

URI : https://id.erudit.org/iderudit/1012241ar

DOI : https://doi.org/10.7202/1012241ar

Aller au sommaire du numéro

\section{Éditeur(s)}

Université du Québec à Montréal

ISSN

0712-8657 (imprimé)

1923-2705 (numérique)

Découvrir la revue

Citer cet article

Majdoub, W. (2011). Médina de Sousse : les enjeux de la gestion touristique d'une ville historique inscrite au patrimoine mondial. Téoros, 30(2), 47-58. https://doi.org/10.7202/1012241ar

\section{Résumé de l'article}

La question de la gestion touristique des sites du patrimoine mondial est en soi complexe. Toutefois, quand le site classé est une médina, cette question devient problématique. Lieux particuliers qui cachent derrière leurs remparts une population pauvre et peu qualifiée, les médinas concentrent à elles seules toutes les problématiques liées au patrimoine et au développement. La lutte contre la pauvreté peut passer par une mise en tourisme et une approche territoriale combinant valorisation culturelle et tourisme durable. Culture, tourisme et développement sont évidemment interreliés. Comment alors inscrire la médina de Sousse dans un processus de gestion touristique durable ? Afin d'y répondre, nous avons étudié deux volets de cette mise en tourisme. L'auteure a voulu comprendre si l'inscription au patrimoine mondial est un label et, en tant que tel, s'il a un effet sur les motivations des touristes pour une destination perçue comme balnéaire malgré sa médina inscrite au patrimoine mondial. Parallèlement, cet article propose une étude des parties prenantes qui sont les marqueurs de la gouvernance et dont la coopération est gage de durabilité.
Ce document est protégé par la loi sur le droit d'auteur. L'utilisation des services d'Érudit (y compris la reproduction) est assujettie à sa politique d'utilisation que vous pouvez consulter en ligne.

https://apropos.erudit.org/fr/usagers/politique-dutilisation/ 


\title{
Médina de Sousse Les enjeux de la gestion touristique d'une ville historique inscrite au patrimoine mondial
}

\author{
Wided MAJDOUB ${ }^{1}$ \\ Maître assistante \\ Université de Sousse, Tunisie \\ Faculté des Sciences Économiques et de Gestion de Sousse \\ wided.majdoub@gmail.com
}

RÉSUMÉ: La question de la gestion touristique des sites du patrimoine mondial est en soi complexe. Toutefois, quand le site classé est une médina, cette question devient problématique. Lieux particuliers qui cachent derrière leurs remparts une population pauvre et peu qualifiée, les médinas concentrent à elles seules toutes les problématiques liées au patrimoine et au développement. La lutte contre la pauvreté peut passer par une mise en tourisme et une approche territoriale combinant valorisation culturelle et tourisme durable. Culture, tourisme et développement sont évidemment interreliés. Comment alors inscrire la médina de Sousse dans un processus de gestion touristique durable? Afin d'y répondre, nous avons étudié deux volets de cette mise en tourisme. L'auteure a voulu comprendre si l'inscription au patrimoine mondial est un label et, en tant que tel, s'il a un effet sur les motivations des touristes pour une destination perçue comme balnéaire malgré sa médina inscrite au patrimoine mondial. Parallèlement, cet article propose une étude des parties prenantes qui sont les marqueurs de la gouvernance et dont la coopération est gage de durabilité.

Mots-clés: Médina de Sousse, label patrimoine mondial, motivations, parties prenantes.

Avec près de $9 \%$ du PIB mondial, le tourisme est en voie de devenir une des premières activités économiques de la planète (WTTC, $2010: 6$ ). Au-delà de cet aspect, il a des répercussions économiques, sociales, identitaires et environnementales qui conduisent à s'interroger sur sa compatibilité avec un développement durable. À côté d'un tourisme de masse «standardisé », on note une évolution vers une forme de consommation plus authentique et porteuse de sens, entraînant un engouement pour la mise en tourisme de zones sensibles ou protégées restées jusque-là relativement préservées (Minvielle et al., 2008 : 19). L'impératif de lutte contre la pauvreté dans ces zones peut passer par une mise en tourisme et une approche territoriale combinant valorisation culturelle et tourisme durable. Culture, tourisme et développement sont interreliés, mais le processus est plus compliqué qu'il n'y paraît. Cette question est particulièrement prégnante dans le cas de la mise en tourisme des médinas. Cet article présente une partie des conclusions d'une étude menée dans le cadre du projet MONTADA portant sur six médinas du Maghreb. L'article se concentre sur le cas de la médina de Sousse. La première partie de ce travail définit les différents concepts utilisés et discute de la place du tourisme culturel dans le processus de réhabilitation des médinas. La question de savoir si l'inscription au patrimoine mondial est un label sera abordée ainsi que les effets d'un tel label. La seconde partie présente la méthodologie en deux volets. Le premier porte sur une analyse quantitative des motivations des touristes. L'auteure a voulu voir si la motivation et le choix de la destination sont liés à l'inscription au patrimoine mondial. Peu d'études ont abordé cette question du point de vue des touristes, mise à part celle de Marcotte et Bourdeau (2006). Le second volet de la méthodologie porte sur l'étude des parties prenantes au travers d'une analyse du discours et d'une étude de la gouvernance.

\section{La médina un lieu référentiel du patrimoine culturel Grandeur et décadence}

Le terme de médina se réfère à la ville historique du sud de la Méditerranée, bien qu'elle soit, comme le souligne Balbo (2010 : 12), un modèle spécifique de ville arabe. Cette notion de "ville historique" est somme toute assez récente. Définitivement formalisée en 1986 par l'ICOMOS 
(International Council on Monuments and Sites), la ville historique se définit comme «les villes, grandes et petites, [...] qui, outre leur qualité de document historique, expriment les valeurs propres aux civilisations urbaines traditionnelles» et sont donc éligibles à la procédure de sauvegarde (ICOMOS, 1986). Pour Abdelquafi (2004: 11), «[1] a doctrine de sauvegarde reconnaît la ville historique comme totalité homogène et cohérente; elle considère le patrimoine monumental et les arts traditionnels comme un héritage matériel et immatériel essentiel à l'épanouissement de l'identité culturelle contemporaine». C'est ainsi que s'est développée l'approche patrimoniale urbaine, qui considère que l'intérêt historique, culturel et esthétique de nombreuses villes ne peut être réduit à la seule présence d'éléments remarquables, mais réside dans l'harmonie et la qualité de l'ensemble des édifices et des espaces qui le composent (Choay, 1992). La médina correspond donc à une notion élargie du patrimoine en tant que trame urbaine de la ville historique, pour peu que les tissus urbains aient gardé une certaine "intégrité». Lieux particuliers qui sont autant de petits noyaux urbains enclavés dans des villes modernes, les médinas cachent derrière leurs remparts une population issue de l'exode rural, pauvre et peu qualifiée (Banque européenne d'investissement, 2009). Ces espaces patrimonialisés (Bouchenaki, 1995) ne cessent d'alimenter l'imaginaire des visiteurs (Amirou, 2000). Les médinas ont pourtant connu ces 50 dernières années une dégradation constante dans l'ensemble de la région du Maghreb, un délabrement matériel et social qui semble inéluctable (UNESCO, 2004). Deux tendances expliquent ce phénomène. En premier, une partie des habitants d'origine abandonne leurs maisons pour aller s'installer dans des logements plus modernes. Les vieilles maisons délaissées par de nombreux héritiers, incapables de s'entendre pour les restaurer, tombent en ruine ou sont louées pour une très modique somme à des familles modestes issues de l'exode rural (El Faïz, 2002). En second, la croissance soutenue des quartiers modernes grignotent peu à peu la vieille ville. En conséquence, la réhabilitation des centres historiques conduit soit à leur requalification, généralement plus commerciale et qui, à terme, dénature le lieu, ou à leur embourgeoisement (Angelini et Godat, 2008). Les remparts cachent donc une réelle paupérisation de la population qui vit dans ce patrimoine et qui a des difficultés à se l'approprier.

\section{Réhabilitation des médinas \\ et tourisme culturel : la panacée?}

Un récent rapport de la Banque mondiale (2010a : 27) souligne pourtant que «les vieilles villes peuvent être un facteur essentiel de continuité et de stabilité : le patrimoine du passé peut venir soutenir le développement de demain ». Ce rapport résume les enjeux : la réhabilitation des médinas pourrait être une source d'enrichissement économique et culturel de ce tissu urbain très complexe. Le tourisme culturel est souvent présenté comme la panacée (Banque mondiale, 2003; Robinson et Picard, 2006; OECD, 2009), segment de marché restreint mais prometteur. Cette niche représente pour le tourisme tunisien une alternative à la masse qui peine toutefois à s'imposer face au monoproduit balnéaire. Comme le souligne le rapport de l'UNESCO (2004), ces villes historiques posent la question du lien qui peut exister entre l'inscription au patrimoine mondial d'une part et leur développement durable d'autre part, au travers d'une patrimonialisation réussie. On peut synthétiser ces enjeux autour de trois volets : la valeur identitaire du patrimoine culturel comme facteur possible de cohésion sociale pour créer un consensus autour des objectifs d'un développement plus durable; le potentiel des activités liées à la sauvegarde et à la valorisation du patrimoine culturel comme vecteur du développement économique, à travers notamment la promotion du tourisme culturel; le défi de composer avec les exigences de la conservation du patrimoine culturel matériel et immatériel et avec les exigences de la modernisation (UNESCO, 2004 : 2). Cette modernisation devrait théoriquement conduire à une amélioration de la qualité de vie. La Banque mondiale (2010b) a récemment élaboré un indice du potentiel touristique d'une médina comme source de croissance économique. Bien que la médina de Sousse réponde aux huit critères, son potentiel touristique est peu ou pas développé. Le cas de la médina de Sousse est édifiant à plus d'un titre. Si la station balnéaire de Sousse draine plus d'un million de touristes par an (ONTT, 2009), la médina regroupant les sites classés semble, elle, boudée par les touristes, ce qui soulève de nombreuses interrogations. En particulier, la perception et la valorisation de l'image de Sousse en tant que ville culturelle pose problème.

\section{Villes historiques, inscription au patrimoine mondial et développement : des relations complexes}

Le tourisme dans les villes historiques est une forme particulière de tourisme culturel (Cazes et Potier, 1998; Van Der Borg, 1998) : à chaque type de villes touristiques correspond une forme de mise en tourisme (Knafou, 2009: 7). Bonard et Felli (2008 : 2) distinguent deux conceptions du tourisme patrimonial urbain : "La première insiste plutôt sur la valeur symbolique et culturelle du patrimoine alors que la seconde met l'accent sur la production du patrimoine dans une logique de valorisation marchande.» Dans la logique de marchandisation, l'authenticité devient la caractéristique première de la «marchandise-patrimoine» (Bonard et Felli, $2008: 5$ ).

Si pour Amirou (2000 : 29) l'imaginaire touristique se caractérise essentiellement par une quête d'authenticité, de quelle authenticité parle-t-on? L'authenticité doit servir alors de pont entre l'offre et la demande touristique (Hamon, 2005 : 44), vers un tourisme plus durable amorçant un cercle vertueux de développement du territoire dans lequel celui-là s'inscrit. La question de savoir si l'inscription au patrimoine mondial est un facteur favorable au développement d'un territoire est loin d'être résolue. Les travaux étudiant les relations entre patrimoine, tourisme et développement sont nombreux (Greffe, 2003; Leask et Fyall, 2006; Peacock et Ilde, 2008; OECD, 2009). D'autres études soulignent, elles, l'impact de l'inscription au patrimoine mondial sur le tourisme à travers une analyse économétrique (Cochrane et Tapper, 2006; Arezki et al., 2009). Il existe par contre moins d'études spécifiques sur la relation entre inscription au patrimoine mondial et développement économique, et l'étude de Gravari-Barbas et Jacquot (2008) offre une synthèse des différents travaux sur la question. Les conclusions de Gravari-Barbas et Jacquot, 
(2008 : 31 et 33 ) montrent qu' «il est difficile de cerner l'importance du patrimoine dans le choix des destinations» et donc de faire la part des choses, principalement parce que «le patrimoine intervient comme un facteur décisif en termes de décision de déplacement vers tel ou tel espace, quand il est soutenu par d'autres facteurs offrant un bien-être plus général». Ce lien semble ténu (Prud'homme, 2008 : 6). D'autres auteurs sont ouvertement plus circonspects. Frey et Steiner (2010:11) ont quant à eux étudié les effets tant positifs que négatifs d'une inscription et proposent une série de trois alternatives à l'inscription d'un site à la Liste du patrimoine mondial : la non-intervention pure et simple, arguant que les sites ne disparaîtront pas; l'utilisation du marché pour une allocation efficace des ressources afin de préserver le patrimoine mondial; l'instauration de listes concurrentes. Pour Siravo (2009), dans le contexte actuel de mondialisation, il est crucial de rallier tous les acteurs sociaux des sites classés afin qu'ils coordonnent leurs efforts dans les processus de préservation et de réhabilitation des villes historiques. Il apparaîtrait que l'inscription d'un site au patrimoine mondial prône des principes paradoxaux : les modes de conservation sont basés sur des critères universels, mais avec des enjeux de développement locaux (Bosredon, 2008). C'est ce qui conduit Lazzarotti (2000: 13) à souligner que "patrimoine et tourisme procèdent d'un même système de valeurs, dont la diffusion mondiale est conforme à un seul et même mouvement de mise en ordre d'un monde». Benhamou (2010 : 129) va encore plus loin dans le raisonnement : "[L]e terme de patrimoine mondial, prometteur, polysémique, repose sur une contradiction. D'un côté, il suppose une insularité du patrimoine en impliquant des normes qui sont au moins en partie extérieures au pays. D'un autre côté, il renvoie à la conservation et à la préservation d'identités culturelles locales.» Cette contradiction la conduit à s'interroger sur «les effets économiques complexes de la labellisation lorsqu'elle ignore le contexte légal, culturel et social du pays» (Benhamou, $2010: 113$ ).

\section{Patrimoine mondial, un label}

La gestion de la destination en tant que marque est un processus complexe. Ayant longtemps évolué séparément, les concepts de destination et de marque convergent aujourd'hui, en témoignent les recherches (Kotler et Gertner, 2002; Gallarza et al., 2002; Balakrishnan et al., 2008; Balakrishnan, 2008; Pike, 2005; Hankinson, 2010) et colloques sur la question publiés récemment (Zenker et Braun, 2010; Braun et Zenker, 2010). Quand les consommateurs ne connaissent pas très bien un produit ou une marque, l'image du pays d'origine permet au consommateur d'identifier les attributs, bénéfices ou symboles du produit à ceux du pays. O'Shaughnessy et O'Shaughnessy (2000) montrent que, de la même manière, une nation peut être considérée comme une marque. Bartikowski et al. (2009) reprennent ce concept pour une ville et développent une échelle de mesure de la personnalité des villes. Pour Botti et al. (2008 : 20), « une destination touristique est bien plus qu'un lieu géographique où le touriste décide de passer du temps. C'est un amalgame de produits, services, ressources naturelles capables d'attirer des visiteurs ». Ce concept est de fait des plus élusifs. Saraniemi et Kylänen (2011 :133), dans une étude du concept de destination, ont identifié quatre approches différentes : 1) un courant de recherche axé sur la géographie économique (Hall et Page, 2006); 2) un courant de gestion basé sur la destination du point de vue marketing (Gunn, 2002; Ritchie et Crouch, 2000; 3); un courant orienté vers le client (Firat et Venkatesh, 1995); et 4) une approche socioculturelle (Ringer, 1998; Saarinen, 2004). Pike (2009 : 861) définit trois principaux courants de recherche : le développement de l'identité de la marque destination, le positionnement de la marque destination et la mesure de la valeur de la marque destination. La marque destination est définie par Zenker et Braun (2010:3) comme « [u]n réseau d'associations dans l'esprit des consommateurs basé sur l'expression visuelle, verbale, et comportementale d'un lieu, qui est incarné par les objectifs, la communication, les valeurs, et la culture générale du lieu». L'étude des labels en marketing a surtout porté sur les produits alimentaires (Giraud, 2001), et les travaux de recherche sur l'influence des labels sur le comportement du consommateur sont assez peu développés. Le label fonctionne comme un ensemble de signaux de qualité de nature informationnelle (Larceneux, 2003 : 37). Le champ du tourisme est un domaine où cette recherche de signal est d'autant plus marquée que le touriste va rechercher des informations sur ces territoires nouveaux (Erwann et Thouément, 2007 : 34). Pour Chameroy et Chandon (2010 : 5), «le label est un signe distinct de la Marque, symbolisant l'une des dimensions intrinsèques du produit, émanant d'une entité tiers ou perçue comme telle». L'effet des labels sur les consommateurs est quant à lui un sujet encore peu débattu. Dans les domaines de l'alimentaire et de l'e-commerce, les recherches montrent que le consommateur a généralement une attitude favorable vis-à-vis des labels et qu'il va en tenir compte lors de la phase de choix, mais, pour qu'un label produise des effets positifs, il faut qu'il combine notoriété, évocations et garantie (Chameroy et Chandon, 2010 : 12; Giraud, 2001). Ces trois éléments nous semblent fondamentaux. Au niveau territorial, l'inscription au patrimoine mondial peut devenir un instrument privilégié de marketing urbain (Courvoisier et Aguillaume, 2011) et, par là même, un outil de communication territoriale dirigé tant vers les populations étrangères au territoire que vers les communautés locales (Noisette et Vallerugo, 1996). Si la destination est une marque, alors on peut considérer l'inscription à la Liste du patrimoine mondial d'un site comme un label. Pour Ryan et Silvanto (2009: 292) le patrimoine mondial est en lui-même une marque. Hall et Piggin (2003) ou encore Marcotte et Bourdeau (2006) montrent que, si label il y a, il n'est toutefois pas suffisant pour convaincre les touristes à choisir la destination.

\section{Label qui conduit à un tourisme culturel durable?}

La gestion touristique des sites du patrimoine mondial est une problématique importante qui pose nombre d'interrogations (UNESCO, 2002) et notamment celle de la durabilité du tourisme culturel tel qu'il est pratiqué dans ces sites emblématiques. Les diverses approches relatives au lien entre tourisme et culture font ressortir les problèmes de définition du tourisme culturel (OECD, 2009 : 22). McKercher et Du Cros (2002:3) regroupent les différentes définitions en quatre catégories : 1) 


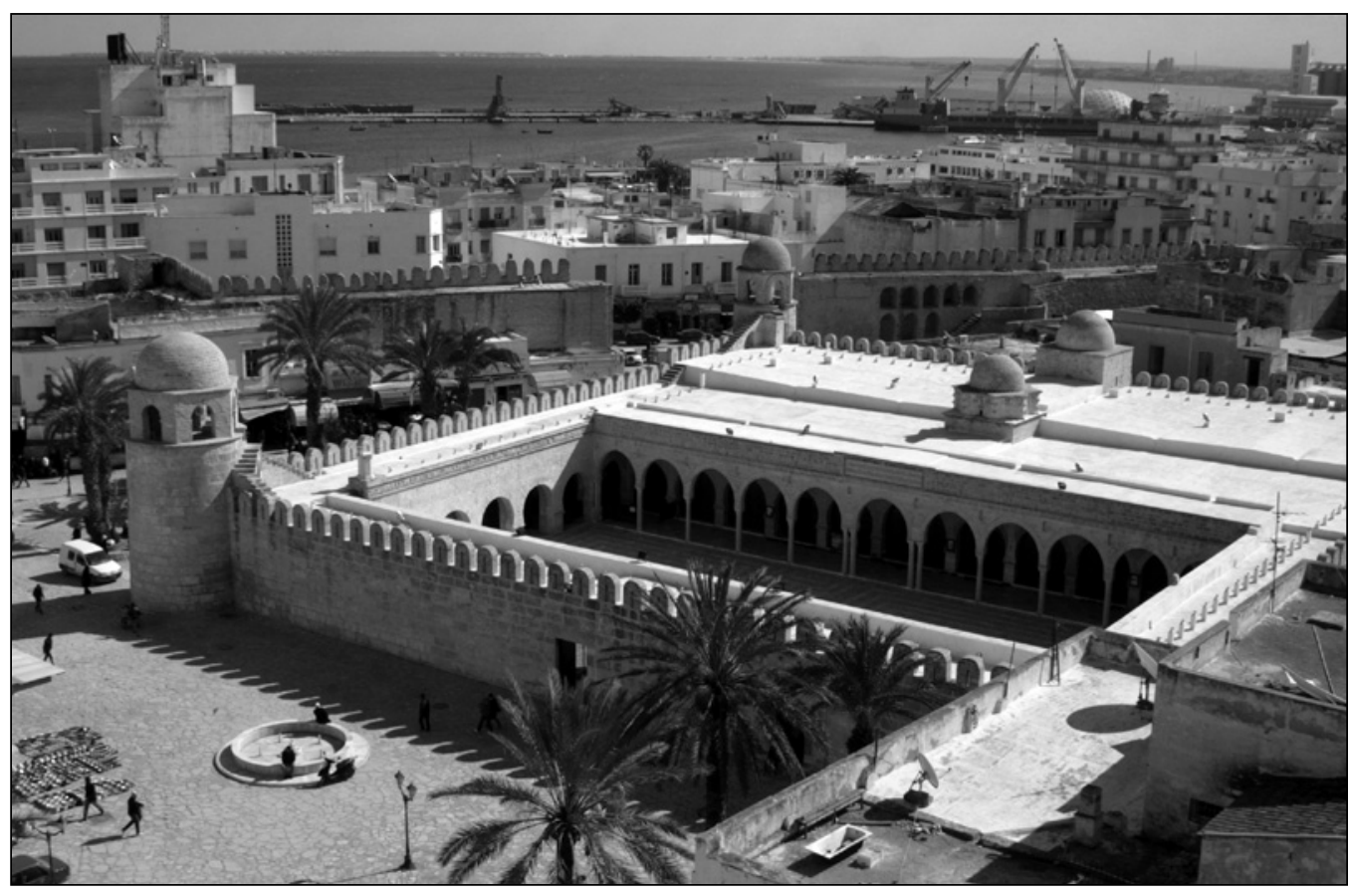

ILLUSTRATION 1 : Une vue de la Grande Mosquée depuis la tour du Ribat (photo : MONTADA). celles découlant du tourisme en tant que concept (McIntosh et Prentice, 1999); 2) les définitions basées sur le motif du voyage (Richards, 1996 et 2002); 3) les définitions basées sur l'expérience (Richards, $2001: 56$ ); et 4) les définitions provenant des organisations internationales (WTO, 1985; ICOMOS, 1999). Nous retrouvons la même position chez Ivanovnic (2009: 77). Lieu commun ambivalent pour Cousin (2006a : 155), le tourisme culturel «est sans doute une invention des institutions internationales». Peut-on en dire de même du tourisme durable? Pour l'OMT (2005: 4), « [i]l y a souvent une certaine confusion concernant le fait que le tourisme durable serait une forme particulière de tourisme attirant un marché étroit, sensible aux impacts environnementaux et sociaux». L'OMT (2005: 5) souligne encore que, «correctement géré, le tourisme de masse peut et devrait être aussi durable que le tourisme à petite échelle, dispersé ou axé sur des centres d'intérêt particuliers». Pour Breton (2009), le tourisme culturel durable est basé sur une gestion appropriée des ressources du patrimoine, et peut constituer un facteur puissant de durabilité et de viabilité. Le label patrimoine mondial peut-il être la clé d'un développement durable? Ou doit-on s'interroger avec Lazzarotti (2010 : 9) sur la question de savoir si le tourisme culturel est durable? Marcotte et Bourdeau (2010 : 283) soulignent l'effet paradoxal de la promotion des sites de la Liste du patrimoine mondial : alors que leur protection augmente, leur attractivité, elle, risque dans le même temps de les détruire. On peut dès lors identifier deux principes clés d'un tourisme culturel durable pour des sites classés au patrimoine mondial : la nécessité pour les destinations de mettre en place un processus de planification à long terme et la participation des parties prenantes dans ce processus (Landorf, 2009). Abdelaas et al. (2005) et Millar (2006) soulignent l'importance de la collaboration des parties prenantes dans la gestion du patrimoine comme gage de durabilité. Dotenwill et Reynaud (2006) abordent la question de la gouvernance des actifs culturels dans une approche par les parties prenantes. Pour Freeman (1984:46), une partie prenante se définit comme "tout individu ou groupe d'individus pouvant affecter l'organisation ou être affecté par la réalisation des objectifs de celle-ci». Dans une perspective de développement touristique durable, trois catégories de parties prenantes peuvent être retenues : les parties prenantes volontaires, qui choisissent de s'associer à l'organisation pour servir leurs intérêts; les parties prenantes involontaires, qui peuvent être affectées (positivement ou négativement) par les activités de l'organisation; et les parties prenantes diffuses, qui peuvent interférer sur la firme de façon externe (Freeman, 1999; Clarkson, 1995). Pour Carrière (2009 : 362), le processus de gouvernance peut être appréhendé selon une politique de gouvernance et une politique de patrimonialisation. Ce processus, on peut l'intégrer comme Flagestad et Hope (2001) et Carrière (2009) dans un cadre plus large, celui de la gouvernance des destinations touristiques.

\section{La médina de Sousse : des enjeux complexes État des lieux et éléments de cadrage}

Sur les 6,5 millions de touristes qui se sont rendus en Tunisie durant l'année 2010, moins de 3 millions auront visité l'un des 14 sites aménagés pour recevoir les visiteurs (leur gestion est confiée à l'Agence de mise en valeur du Patrimoine et de la Promotion Culturelle - AMVPPC par la suite). La Tunisie a fait dès les années 1960 le choix du tourisme balnéaire, peu spécifique, et caractéristique du tourisme de masse, avec toutes les conséquences que cela implique (économiques, sociales et environnementales). Entre les années 1980 et 1990, la Tunisie a développé les sites balnéaires. Les efforts de diversification n'ont été entrepris que récemment. Nous assistons depuis 
2007 à une évolution du profil de la clientèle. Le tourisme tunisien est traditionnellement très dépendant des marchés européens. Allemands, Français et Italiens comptaient pour $48 \%$ de l'ensemble des visiteurs en 2000 , et pour $65 \%$ des nuitées (ONTT, 2009). Ces chiffres ont chuté à 56,9\% en 2010, en raison d'une concurrence internationale accrue et d'un attrait moindre pour la destination. En revanche, depuis dix ans, la Tunisie réussit à attirer un plus grand nombre de visiteurs en provenance d'Afrique du Nord (Libyens et Algériens) : ceux-ci représentaient $37 \%$ du nombre total des visiteurs en 2006, contre aujourd'hui $40,9 \%$ des arrivées des non-résidents en Tunisie avec 2,651 millions de visiteurs comptabilisés aux frontières (ONTT, 2009). Le rapport de la Banque mondiale (2002) préconisait le développement de nouvelles filières qui pourraient apporter un avantage compétitif au tourisme tunisien. À l'heure actuelle, face aux difficultés du secteur et compte tenu de la conjoncture internationale, la stratégie s'est tournée vers la diversification du produit touristique. Modèle balnéaire et modèles alternatifs ne s'opposent pas, mais l'on observe une diversification et un enrichissement d'un tourisme balnéaire qui reste incontournable. La région de Sousse est tout à fait représentative de ce type de tourisme : avec 120 hôtels, sa capacité est de 40000 lits (ONTT, 2009). La médina de Sousse est donc une ville historique enclavée dans un cadre balnéaire (voir illustration 1).

\section{Ville historique}

Avec une superficie de 31 hectares et 7000 habitants, la médina de Sousse a vu cinq de ses monuments inscrits depuis 1988 à la Liste du patrimoine mondial. Avec le Ribat, la Kasbah, les remparts, la mosquée de Bu Ftata et la Grande Mosquée, Sousse apporte un témoignage exceptionnel sur la civilisation des premiers siècles de l'Hégire. Le plus ancien et le mieux conservé de toute la série, le Ribat de Sousse, est un exemple éminent de ce type de construction (voir illustration 2). La médina dans son ensemble constitue un exemple éminent d'habitat humain traditionnel devenu vulnérable sous l'effet de mutations irréversibles.

L'histoire du lieu marque sa valeur exceptionnelle et constitue l'un des critères de son inscription. C'est son infrastructure et son architecture typiques qui font l'identité du site : pas moins de six grandes portes historiques permettent l'accès en son sein. La médina compte 59,8\% de maisons traditionnelles (12,9\% de maisons bourgeoises, 45,6\% de maisons modestes, 41,5\% de maisons populaires) (ARRU, 2010). Certaines ont été restaurées et sont depuis aménagées en musées (voir illustration 3).

Les statistiques de l'AMVPPC (nombre d'entrées aux monuments avec entrée payante : Grande Mosquée, Ribat, Musée archéologique) permettent d'évaluer la demande touristique de la médina. On considère après enquête que tout visiteur ayant visité le Ribat a visité en conséquence une partie au moins de la médina. Ainsi, pour Sousse, le nombre total des visiteurs des trois sites inscrits pour la Grande Mosquée, Ribat et la médina (le Musée est fermé pour rénovation depuis 2008) est en régression, tandis que les chiffres pour la ville de Sousse en tant que destination sont stabilisés autour du million de touristes sur les quatre dernières années (voir tableau 1). Sur

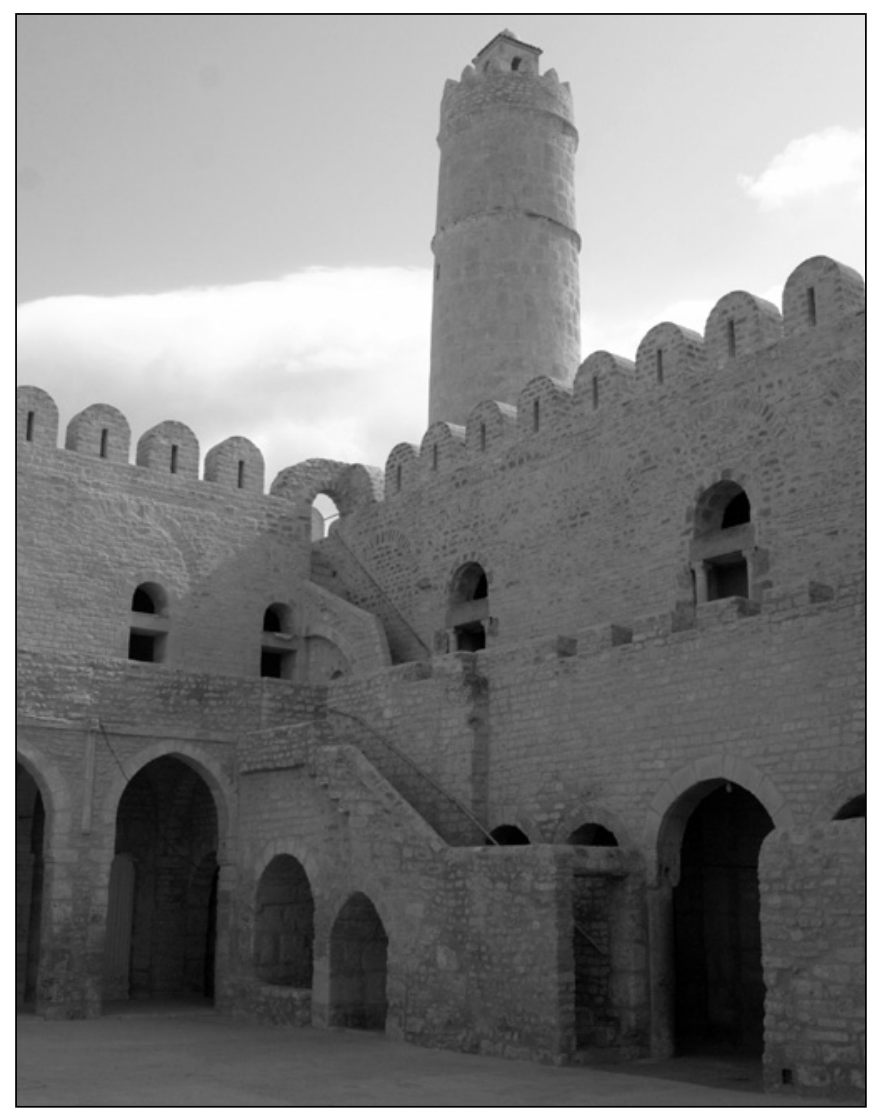

ILLUSTRATION 2 : Une vue intérieure de la cour du Ribat (photo : MONTADA).

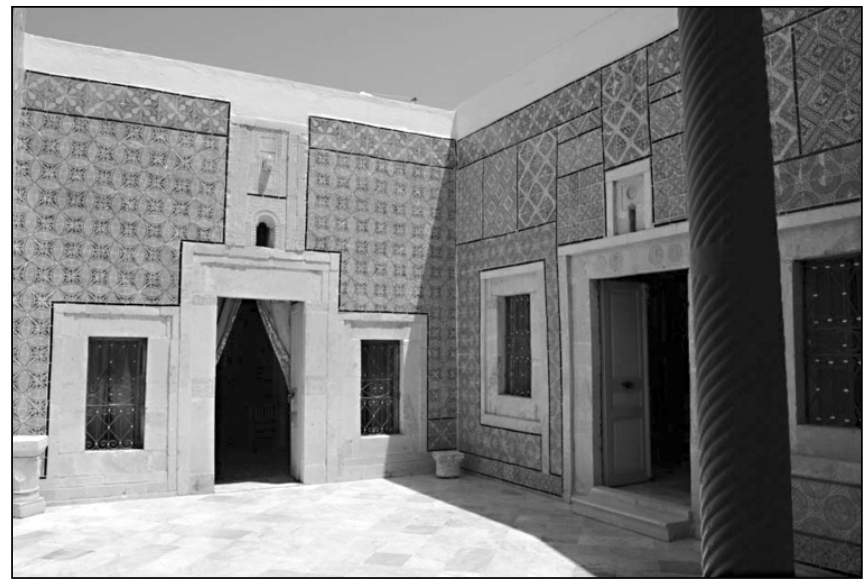

ILLUSTRATION 3 : Dar Essid, le patio d'une maison traditionnelle transformée en musée privé (photo : MONTADA).

le million de visiteurs en 2009, 75271 ont visité la médina, soit près de $7 \%$ du total de la ville de Sousse (ONTT, 2009). Il est important de souligner qu'il s'agit, pour la quasi-totalité, de visites inclues dans le séjour par les voyagistes et, pour une infime partie, de prestations proposées par les agences de voyage locales à travers les hôteliers. 
Tableau 1: Entrées payantes aux monuments et données du séjour pour la ville de Sousse

\begin{tabular}{c|c|c|c|c|c}
\hline & Grande Mosquée & Musée & Ribat & Médina & Sousse ville \\
\hline 2000 & 86851 & 54936 & 118438 & 260225 & 984469 \\
\hline 2001 & 77851 & 49266 & 116247 & 243364 & 965812 \\
\hline 2002 & 50530 & 38644 & 75530 & 164704 & 885562 \\
\hline 2003 & 45752 & 33800 & 74503 & 154055 & 902164 \\
\hline 2004 & 53391 & 37440 & 80446 & 171277 & 981287 \\
\hline 2005 & 50805 & 40786 & 108821 & 200412 & 1050766 \\
\hline 2006 & 50081 & 35216 & 88424 & 173721 & 1039991 \\
\hline 2007 & 52104 & 12665 & 79962 & 144731 & 1086991 \\
\hline 2008 & 48947 & 0 & 64779 & 113726 & 1055646 \\
\hline 2009 & 29113 & 0 & 46158 & 75271 & 1006439 \\
\hline
\end{tabular}

Source : compilation de l'auteure à partir des données de base de l'AMVPPC (2009) et de l'ONTT (2009).

Tableau 2: Profil sociodémographique de l'échantillon de l'étude

\begin{tabular}{c|c|c|c}
\hline \multicolumn{2}{c|}{ Âge } & \multicolumn{2}{c}{ Sexe } \\
\hline Entre 20 et 29 ans & 53 & Féminin & 47 \\
\hline Entre 30 et 39 ans & 14 & Masculin & 53 \\
\hline Entre 40 et 49 ans & 21 & Niveau d'instruction \\
\hline Entre 50 et 59 ans & 9 & Primaire & 4.5 \\
\hline Entre 60 et 69 ans & 2 & Secondaire & 20 \\
\hline Plus de 69 ans & 1 & Universitaire & 75,5 \\
\hline
\end{tabular}

Source : enquête effectuée sous la direction de l'auteure auprès d'un échantillon de 135 personnes, de juillet à aout 2010 .

\section{Tableau 4: Fréquentation pour l'échantillon des différents sites}

\begin{tabular}{|c|c|}
\hline \multicolumn{2}{|c|}{$\%$ des personnes ayant visité les principaux monuments de Sousse } \\
\hline Le musée Dar Essid (dans l'enceinte de la médina) & 10,7 \\
\hline La Kobba (dans l'enceinte de la médina) & 11,6 \\
\hline Les Catacombes (en dehors de la médina) & 4,1 \\
\hline L'église Saint-Félix (en dehors de la médina) & 7,4 \\
\hline La synagogue (en dehors de la médina) & 7,4 \\
\hline \multicolumn{2}{|c|}{ \% des personnes ayant visité les sites classés de la médina } \\
\hline La médina & 73,6 \\
\hline Le Ribat & 19,8 \\
\hline La Grande Mosquée & 27,3 \\
\hline
\end{tabular}

Ville historique enclavée dans un cadre balnéaire L'enjeu de la présente étude se précise : si l'inscription au patrimoine mondial est un label, il serait intéressant d'en étudier les effets tant pour les touristes que pour les communautés locales qui vivent dans ces médinas inscrites. Nous avons voulu comprendre en premier lieu les raisons de cette apparente désaffection et mené une enquête auprès d'un
Tableau 3: Sousse en tant que destination touristique

\begin{tabular}{|c|c|c|c|}
\hline \multicolumn{2}{|c|}{$\begin{array}{l}\text { Connaissance de l'inscription de } \\
\text { la médina au patrimoine mondial }\end{array}$} & \multicolumn{2}{|c|}{$\begin{array}{l}\text { Considérez-vous Sousse comme } \\
\text { une destination culturelle? }\end{array}$} \\
\hline Oui & 29 & Oui & 48 \\
\hline Non & 71 & Non & 52 \\
\hline \multicolumn{4}{|c|}{ Principales attractions de la ville de Sousse } \\
\hline La plage & \multicolumn{3}{|c|}{83,3} \\
\hline Les loisirs & \multicolumn{3}{|c|}{30} \\
\hline La vie nocturne & \multicolumn{3}{|c|}{27.5} \\
\hline La culture & \multicolumn{3}{|c|}{15,8} \\
\hline \multicolumn{3}{|c|}{$\begin{array}{l}\text { Les éléments de l'image de Sousse en tant que } \\
\text { destination, réponses "s'applique très bien" }\end{array}$} & $\%$ \\
\hline \multicolumn{3}{|c|}{ Destination où l'on peut facilement sortir, s'amuser, faire la fête } & 17,7 \\
\hline \multicolumn{3}{|c|}{ Nourriture de bonne qualité } & 23,4 \\
\hline \multicolumn{3}{|c|}{ Destination culturelle } & 12,2 \\
\hline \multicolumn{3}{|c|}{ Qualité des services } & 22,3 \\
\hline \multicolumn{3}{|c|}{ Destination à la mode } & 25,6 \\
\hline \multicolumn{3}{|c|}{ Hébergement de bonne qualité } & 35,5 \\
\hline \multicolumn{3}{|c|}{$\begin{array}{c}\text { Destination sûre du point de vue de la sécurité des biens } \\
\text { et des personnes }\end{array}$} & 52,2 \\
\hline \multicolumn{3}{|c|}{ Bon rapport qualité-prix du séjour } & 28,9 \\
\hline \multicolumn{3}{|c|}{ Hospitalité de la population locale } & 47,8 \\
\hline
\end{tabular}

Source : enquête effectuée sous la direction de l'auteure auprès d'un échantillon de 135 personnes, de juillet à aout 2010 .

échantillon de 135 touristes de Sousse durant la période de juillet à août 2010 (voir tableau 2).

Le tableau 3 présente les principaux résultats de l'enquête. On y apprend que Sousse n'est pas perçue comme une destination culturelle (pour $52 \%$ de l'échantillon interrogé), la motivation principale étant le balnéaire, et ce, pour 83,3\% des personnes interrogées. Seuls $15,8 \%$ ont une motivation 
culturelle et, pour notre échantillon, seuls $29 \%$ des touristes interrogés avaient connaissance du fait que la médina de Sousse était inscrite au patrimoine mondial. Ces touristes que l'on peut qualifier de culturels sont minoritaires pour notre étude. Ces éléments sont corroborés par les résultats des éléments de l'image de Sousse : elle n'est pas perçue comme une destination culturelle $(12,2 \%)$, mais plutôt comme une destination sûre, d'un bon rapport qualité-prix.

Concernant l'information touristique, on peut la considérer comme quasiment inexistante. Il est très difficile pour les visiteurs d'obtenir des informations écrites concernant les attractions, services ou infrastructures touristiques de la ville. Il n'existe en effet pas de brochures concernant l'offre touristique de la médina (ni d'ailleurs pour la ville de Sousse) en termes de monuments, sites à visiter ou encore d'hébergements, restauration, commerces. Si quelques panneaux d'orientation ont récemment été placés en certains points de la médina, il reste difficile pour les visiteurs de simplement les repérer et de s'orienter dans l'entrelacement des ruelles Cette signalétique qui reprend le circuit en miniature sur des petites plaques en faïences et qui indiquent l'endroit où se situe le visiteur par rapport au circuit est de fait illisible de par ses dimensions (trop réduites) ou l'environnement dans laquelle la plaque s'insère (voir illustration 4).

Le fait de visiter la médina ne signifie pas pour autant visiter tous les sites classés. Pour la grande majorité des cas, il s'agit de traverser les souks et les ruelles marchandes dans le but de faire des achats. Ainsi sur les 73,6\% des touristes qui déclarent avoir visité la médina, seuls $19,8 \%$ ont visité le Ribat, 27,3 \% la Grande Mosquée et 10,7 \% uniquement le musée Dar Essid (voir tableau 4).

Ces chiffres sont corroborés par les données recueillies auprès de l'AMVVPC pour le nombre d'entrées payantes aux monuments classés de la médina. Le label patrimoine mondial n'a que peu d'effet sur l'image de la ville de Sousse, faute sans doute de marketing urbain ou, comme le souligne Rosemberg (1997), faute de discours cohérent intervenant dans la construction du projet de la ville. Le rôle du marketing urbain est donc de développer une image de la ville, son identité, afin de permettre à la ville «de «se positionner» sur «le marché des villes», en «vendant» des réalisations et une image» (Rosemberg, $2000: 1$ ). Un tel projet manque cruellement dans le cas de la ville de Sousse, sans doute parce qu'il doit avant tout fédérer et impliquer toutes les parties en présence.

\section{Compréhension de la dynamique des relations entre les parties prenantes}

L'importance des parties prenantes dans le tourisme en général et dans la gouvernance des actifs culturels est soulignée par de nombreux travaux (Flagestad et Hope, 2001; Yilmaz et Gunel, 2009; ou Carrière, 2009). Freeman (1984) aborde la gouvernance des parties prenantes en distinguant trois niveaux : 1 ) le niveau rationnel qui consiste à repérer les parties prenantes et leurs intérêts respectifs; 2) le niveau inhérent aux processus permettant de prendre en compte les intérêts des parties prenantes dans les programmes d'élaboration, d'application et de contrôle de la stratégie mise en œuvre; et 3) le niveau

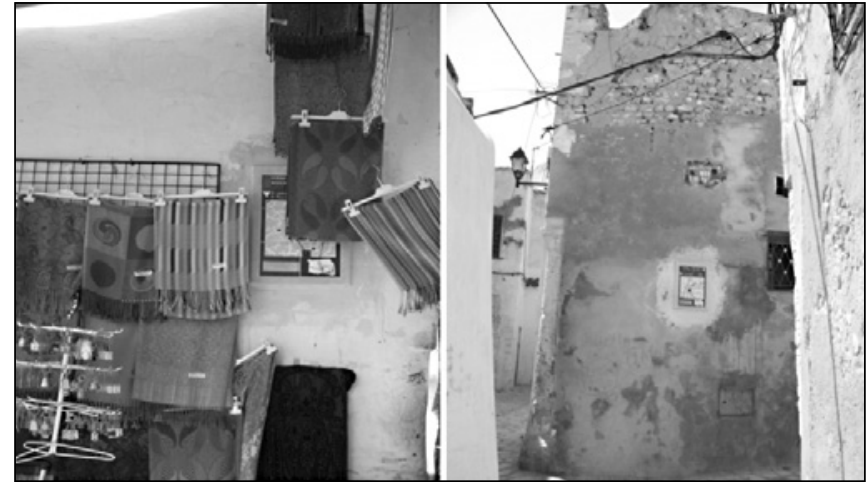

ILLUSTRATION 4 : Une signalétique non adaptée (photo : MONTADA).

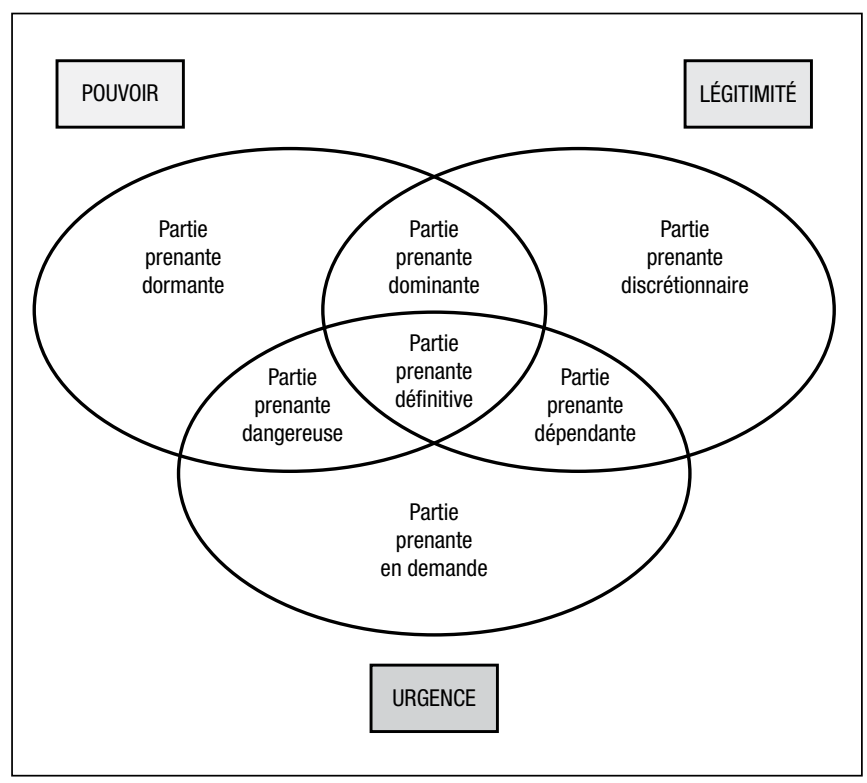

ILLUSTRATION 5 : Typologie des parties prenantes

(source : schéma traduit de l'anglais, Mitchell et al. (1997 : 874)).

des transactions lié aux ressources et aux moyens mobilisés pour gérer les interactions entre l'organisation et ses parties prenantes. Il en découle que la manière dont l'organisation imbrique ces trois niveaux d'analyse influe indéniablement sur la qualité de la gouvernance. La gouvernance est donc, selon Freeman (1984), liée à la manière dont sont agencées les relations entre les parties prenantes. Notre premier objectif a été d'identifier les parties prenantes liées à la médina de Sousse pour ensuite analyser leurs intérêts respectifs, en termes de convergence/divergence, selon la méthode développée par Friedman et Miles (2006 : 28-29).

Le secteur public est représenté par les instituts et établissements publics administratifs et non administratifs qui sont sous tutelle des ministères intervenants dans la gestion de la médina. Ce secteur comprend le Gouvernorat de Sousse, l'arrondissement municipal de la médina (Service de sauvegarde de la médina et des monuments historiques et religieux), l'Agence de la réhabilitation et de la rénovation 


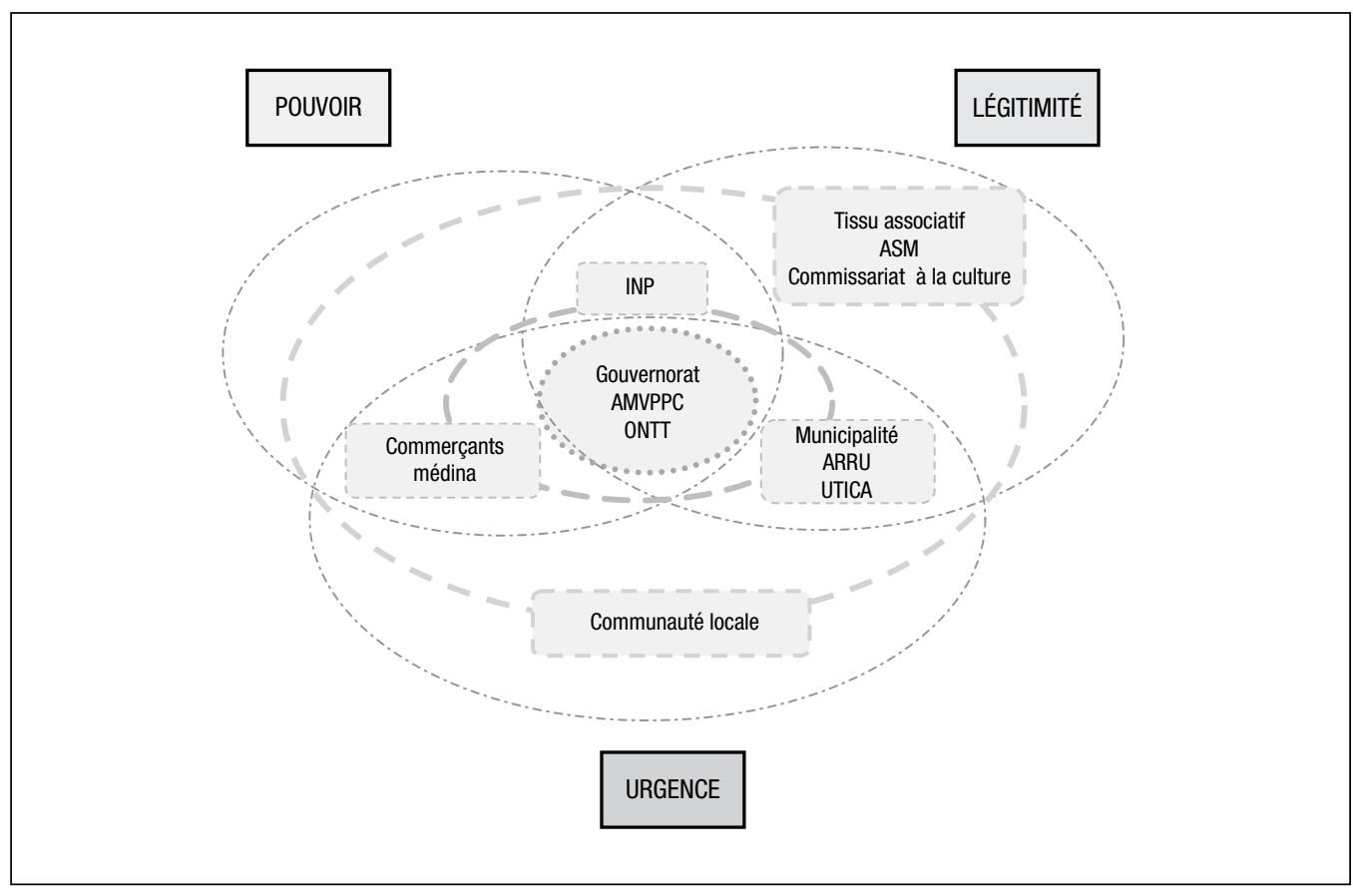

ILLUSTRATION 6 : Une typologie des parties prenantes de la médina de Sousse (source : adapté de Mitchell et al. (1997: 874)).

urbaine (ARRU), l'Institut national du patrimoine (INP), l'Agence de mise en valeur et de la Promotion du Patrimoine Culturel (AMVPPC), le Commissariat à la culture et à la sauvegarde du patrimoine, l'Office national du tourisme tunisien (ONTT). Comme instance syndicale, citons l'Union tunisienne des industriels commerçants et artisans (UTICA). Le secteur associatif comprend l'Association de la sauvegarde de la médina de Sousse (ASM), l'Association des recherches et des études de la médina de Sousse (AREMS), la Société archéologique de Sousse (SAS) et l'Association de sauvegarde des arts authentiques (ASAA). Le secteur privé comprend, lui, les hôteliers et les agences de voyages, ou encore les musées privés (Dar Essid et Dar Baba). Enfin, il y a la communauté locale qui vit dans ce patrimoine. L'étude, sous forme d'étude de cas, s'est faite en trois temps avec en premier une étude des documents d'archive et leur analyse. Puis nous avons rencontré les différents responsables et analysé leur discours. Enfin, après avoir listé toutes les parties prenantes, nous avons construit une typologie basée sur celle de Mitchell et al. (1997 : 872-873) reposant sur l'identification de trois critères de pertinence des parties prenantes : le pouvoir, la légitimité et l'urgence (voir illustration 5).

N'ayant ni légitimité ni demande urgente, la partie prenante est dite dormante. La partie prenante discrétionnaire n'a ni pouvoir d'influence ni demande urgente. La partie prenante en demande est revendicatrice mais elle n'a ni pouvoir ni légitimité. La partie prenante dominante possède deux des attributs les plus importants : le pouvoir et la légitimité. La partie prenante dépendante exprime, elle, des demandes légitimes urgentes mais n'a pas le pouvoir nécessaire. La partie prenante dangereuse a des demandes urgentes mais illégitimes et peut abuser de son pouvoir. Quant à la partie prenante définitive, elle possède les trois attributs.

\section{Gouvernance en panne}

D'après la théorie de l'identification et de la prégnance des parties prenantes (Mitchell et al., 1997 : 874), nous pouvons classer les parties prenantes en trois catégories selon les trois critères de pertinence (le pouvoir, la légitimité et l'urgence). L'illustration 6 récapitule les combinaisons de ces trois critères qui définissent trois grandes familles de parties prenantes : celles qui font autorité, celles en position d'attente et enfin les parties prenantes latentes.

Si nous raisonnons par cercles concentriques, à l'intersection de ces trois critères, nous trouvons trois parties incontournables du secteur public : décisives, elles font autorité. Le gouvernorat exerce un contrôle direct sur toutes les décisions et joue le rôle d'intermédiaire actif entre la municipalité et les autres preneurs de décisions à l'échelle régionale et nationale. L'AMVPPC a été critiquée par plusieurs parties prenantes. Premier bailleur de fonds pour les interventions de restauration et réhabilitation de la médina, l'agence gère les fonds des entrées payantes des monuments. C'est une partie peu ouverte. L'ONTT est un des principaux bailleurs de fonds pour le financement de certains projets de réhabilitation de la médina, très passif sur le plan communicationnel, malgré ses prérogatives. Décisives, ces parties prenantes sont dans notre cas précis déconnectées les unes des autres.

Le second cercle se définit par la présence de deux des trois critères, les parties prenantes sont dites en position d'attente. On distingue :

- les dominantes : Par exemple, l'INP a le pouvoir et la légitimité d'agir. Pourtant on note un manque notoire de coopération avec les autres parties prenantes, ce qui lui conferre une certaine inertie;

- les dépendantes : La municipalité de la médina de Sousse manque cruellement d'experts, et notamment de 
techniciens, d'architectes, d'urbanistes, de sociologues. L'ARRU est un simple opérateur auprès de la municipalité, sans véritable pouvoir d'action. L'UTICA représente les commerçants en activité. Les rapports avec les autres parties prenantes sont souvent conflictuels;

- et les dangereuses : Dans le secteur privé, nous distinguons les créateurs privés (on compte dans la médina deux musées privés : Dar Essid et Dar Baba) des commerces, boutiques d'artisanat et souks. Les premières parties prenantes sont dites revendicatrices. Le commerce dans la médina prend actuellement une nouvelle forme : on assiste à une augmentation importante des boutiques des faux articles d'artisanat où la plupart des produits sont fabriqués en Chine, et à la disparition des métiers d'arts et d'artisanat typiques et authentiques qui la caractérisaient. Ce type de commerces attire certes les touristes pour acheter des souvenirs à bas prix, mais véhiculent aussi une perte d'authenticité et une expérience négative car teinte d'une forte agressivité (Majdoub, 2011).

Dans le dernier cercle, les parties prenantes ne possèdent qu'un seul des trois attributs. Ces parties prenantes sont dites latentes. Quelques-unes ont une certaine légitimité. C'est le cas du tissu associatif regroupant l'ASM, l'AREMS, la SAS et l'ASAA. Ces associations different par le niveau de volonté et de conscience de la valeur du patrimoine, de leur volonté de sauvegarde et de leur ouverture au public. Il existe un manque flagrant de collaboration et de coopération entre toutes les associations, qui au mieux s'ignorent. Ce secteur associatif, resté trop longtemps discrétionnaire, est pourtant un acteur privilégié pour faire évoluer les mentalités et encourager l'expression citoyenne. La dernière catégorie, la communauté locale, est une partie prenante revendicatrice mais dont la voix a du mal à se faire entendre. Peu impliquée, la population locale a du mal à s'approprier le patrimoine dans lequel elle vit au quotidien.

Ces différents cercles concentriques se superposent les uns aux autres en différentes couches, et les parties prenantes ont du mal à coopérer ou simplement à communiquer entre elles. Ces acteurs ne partagent pas les mêmes objectifs en termes de gestion touristique, s'ignorent mutuellement et, quand ils se rencontrent, mettent en avant le non-respect de leurs engagements mutuels. Cette situation est symptomatique de l'absence d'un objectif commun porteur d'un projet global. La situation de la médina est critique, car aucun acteur n'est capable de définir ni de mettre en œuvre un projet collectif. Cette situation est problématique pour une destination touristique, comme le montre Van der Yeught (2009 : 313) lorsqu'elle souligne que "l'émergence d'un acteur stratégique est nécessaire au succès des destinations recherchant un développement durable». Nous sommes loin du modèle communautaire de Flagestad et Hope (2001 : 452) dans lequel les différentes parties prenantes doivent collaborer entre elles. On aurait pu croire que l'existence de l'inscription au patrimoine mondial aurait pu constituer un projet collectif. Ce n'est pas le cas. Benhamou (2010 : 12) souligne pour un autre site (Luang Prabang, au Laos) les effets pervers de l'inscription au patrimoine mondial de l'humanité : l'introduction d'un corpus étranger de normes qui les rend difficilement applicables. Il faut donc distinguer, à l'instar de Cousin (2006a : 27), « le tourisme comme secteur économique du tourisme culturel comme valeur politique et identitaire». Est-ce à dire que l'on ne peut pas promouvoir un développement touristique raisonné et durable fondé sur la valorisation du patrimoine culturel matériel et immatériel de la médina? Il faut pour cela que les populations locales soient plus impliquées et qu'elles s'approprient tant le patrimoine dans lequel elles vivent que les retombées économiques de la mise en tourisme de la médina. Pour l'UNESCO (2006), il est important d'intégrer le patrimoine culturel des villes dans les politiques de développement. Cependant, ceci ne peut se faire dans les médinas qu'à la condition que les requalifications urbaines soient basées sur une action multiforme de réhabilitation du cadre de vie des communautés locales. La mise en tourisme des médinas peut alors, au travers de la valorisation du patrimoine, devenir une source de notoriété pour la ville. Elle peut aussi favoriser une forme de développement touristique durable tout en suscitant également dans la population un sentiment d'appartenance à la ville en favorisant une appropriation par les habitants du patrimoine qu'ils habitent (OEDC, 2009). Interpréter et communiquer la valeur de la médina en tant que patrimoine mondial de l'humanité permettraient d'assurer le financement nécessaire au bon entretien et à la restauration des habitats et édifices anciens et abandonnés.

\section{Conclusion}

La mise en tourisme des médinas pose une double question : celle de l'identité (et de l'appropriation par les communautés locales de cette identité et de ce patrimoine) mais aussi celle du «bon tourisme»(Picard, 2001 : 112). Cette étude montre que Sousse, en tant que ville historique, n'a pas une image de ville culturelle. Le tourisme qui y est pratiqué est un tourisme de masse, bien loin de ce «bon tourisme, bon pour les territoires et les populations, bon pour les touristes, et bon pour les organismes qui le promeuvent» (Cousin, 2006b : 16). À quelles conditions le tourisme peut-il jouer un rôle dans la patrimonialisation des médinas, surtout celles classées au patrimoine mondial? Cette étude montre une fois de plus la difficulté d'établir la plus-value de l'inscription au patrimoine mondial. Le lien entre inscription et choix de la destination est ténu et peu significatif dans notre cas d'espèce. Cela est vrai tant sur le plan des motivations des touristes que sur celui de la communication de la destination et de son marketing territorial. La question de la communication autour de l'inscription des sites du patrimoine mondial est loin d'être simple et nous conduit à nous interroger avec Marcotte et Bourdeau (2010 : 279) sur le rôle des parties prenantes pour assurer à la fois la protection des sites et le développement de l'industrie touristique. D'après notre étude, cela ne peut se faire qu'à travers une gestion touristique concertée, sur la base d'un projet de la ville impliquant toutes les parties prenantes. La volonté politique de promouvoir et d'encourager l'investissement de projets à vocation patrimoniale et culturelle est une condition sine qua non. À l'heure actuelle, cette rigidité représente le principal obstacle à une gestion durable du site de la médina. En effet, le 
compromis semble essentiel afin de composer avec les désaccords des parties prenantes (Lorrain, $2000: 38$ ). C'est à cette seule condition que l'on peut avoir une meilleure gouvernance de la médina et, par la même occasion, une gestion et un projet de développement d'un site qui instaure un cercle vertueux de patrimonialisation.

\section{Note}

1 L'auteure souhaite remercier N. Ben Salem, étudiante, qui a été chargée d'identifier les différentes parties prenantes.

\section{Références}

ABDELAAS, Christina; Adele LADKIN et John FLETCHER (2005)

"Stakeholder collaboration and heritage management », Annals of

Tourism Research, vol. 32, n 1, p. 28-48.

ABDELQUAFI, Jallal (2004) La dimension patrimoniale de la ville historique et le cadre institutionnel de la sauvegarde, rapport UNESCO : Patrimoine et développement durable dans les villes historiques du Maghreb contemporain : enjeux, diagnostics et recommandations, rapport final de la rencontre "Fès 2003», coordination scientifique D. Pini, Rabat : Bureau de l'UNESCO à Rabat, p. 7-21.

AMIROU, Rachid (2000) Imaginaire du tourisme culturel, Paris : Presses Universitaires de France. 155 p.

ANGELINI, Sara et Ivan GODAT (2008) «Un exemple d'acteurs de la gentrification : Les propriétaires de maison d'hôtes ", La gentrification dans la médina de Marrakech, Neuchâtel : Université de Neuchâtel, Institut de Géographie, p. 25-48. <http://www2.unine.ch/files/content/sites/ inst_geographie/files/shared/documents/marrakech2008terrainfinal. pdf $>$, consulté en mars 2010.

AREZKI, Rabah; Reda CHERIF et John PIOTROWSKI (2009) «Tourism Specialization and Economic Development: Evidence from the UNESCO World Heritage List ", International Monetary Fund Working Paper, WP/09/176, Washington : International Monetary Fund. 25 p.

ARRU - AGENCE DE LA RÉHABILITATION ET DE LA RÉNOVATION URBAINE (2010) Projet d'intervention sur la Médina : première phasel avant-projet sommaire, rapport ARRU, avril, $35 \mathrm{p}$.

BALAKRISHNAN, Melodena Stephens (2008) «Dubai - A star in the east: A case study in strategic destination branding», Journal of Place Management and Development, vol. 1, $\mathrm{n}^{\circ}$ 1, p. 62-91.

BALAKRISHNAN, Melodena Stephens; Ramzi NEKHILI et Clifford LEWIS (2008) «Destination Brand Components», Working Paper Series, WP69/2008, 15 p. $<$ http://ro.uow.edu.au/cgi/viewcontent.cgi?article $=10$ $12 \&$ context $=$ dubaiwp $>$, consulté en novembre 2010.

BALBO, Marcello (2010) «Méditerranée à venir, avenir des médinas", chapitre 1 dans Médinas 2030 - Scénarios et stratégies, p. 11-20. Paris : L'Harmattan.

BANQUE EUROPÉENNE D'INVESTISSEMENT (2009) Médinas 2030 : séminaire organisé dans le cadre de la Semaine Économique de la Méditerranée (Marseille, France : 7 au 10 octobre 2009), <http://www. eib.org/projects/events/seminar-on-mediterranean-cities.htm>, consulté en octobre 2010.

BANQUE MONDIALE (2002) «Stratégie de développement touristique en Tunisie», rapport de la Banque mondiale, phase 1, juillet, $165 \mathrm{p}$.

BANQUE MONDIALE (2003) «Patrimoine culturel et développement», cadre d'action pour le Moyen-Orient et l'Afrique du Nord, Washington : Banque mondiale. $126 \mathrm{p}$.

BANQUE MONDIALE (2010a) The Urban Rehabilitation of Medinas: The World Bank Experience in the Middle East and North Africa, rédigé par
Anthony G. BIGIO et Guido LICCIARDI. Washington : The World Bank, Urban Development and Local Government Unit. 40 p.

BANQUE MONDIALE (2010b) «Indice de potentiel touristique d'une médina (MTPI) », Moyen-Orient et Afrique du Nord, <http:// go.worldbank.org/8XHAQ7UNP0>, consulté en juin 2010.

BARTIKOWSKI, Boris; Dwight MERUNKA; Abdoulaye OUATTARA et Pierre VALETTE-FLORENCE (2009) «Les villes ont-elles une personnalité?», Revue Française de Gestion, vol. 7, nº 197, p. 49- 64.

BENHAMOU, Françoise (2010) «L'inscription au patrimoine mondial de l'humanité : La force d'un langage à l'appui d'une promesse de développement», Revue Tiers Monde, n² 202, p. 113-130.

BONARD, Yves et Romain FELLI (2008) «Patrimoine et tourisme urbain. La valorisation de l'authenticité à Lyon et Pékin ", Articulo - revue de sciences humaines, p. 1-12. <http://articulo.revues.org/719>, consulté en janvier 2010.

BOSREDON, Pauline (2008) «Comment concilier patrimonialisation et projet urbain? Le classement au Patrimoine Mondial de l'Unesco de la vieille ville de Harar (Éthiopie)», Autrepart, n 47, p. 125-147.

BOTTI, Laurent; Nicolas PEYPOCH et Bernardin SOLONANDRASANA (2008) Ingénierie du tourisme : Concepts, méthodes, applications, coll. « Les métiers du tourisme», Bruxelles : Éditions De Boeck. 167 p.

BOUCHENAKI, Mounir (1995) «L'UNESCO et la sauvegarde du patrimoine urbanistique et architectural des Médina», Madina, cité du monde, $\mathrm{n}^{\circ} 1$, Paris, p. 22-25.

BRAUN, Erik et Sebastian ZENKER (2010) «Towards an Integrated Approach for Place Brand Management», Proceedings 50th European Regional Science Association Congress (Jönköping, Sweden :

19-23 août 2010), <http://www.fek.su.Se/Global/Forskning/Seminarier/ Braun_Zenker\%20\%20Towards\%20an\%20Integrated\%20Approach\%20 for\%20Place\%20Brand\%20Management.pdf >, consulté en octobre 2010.

BRETON, Jean-Marie (dir.) (2009) Patrimoine culturel et tourisme alternatif: (Afrique, Amériques, Caraïbe, Europe), Paris : Éditions Karthala. 416 p.

CARRIÈRE, Jean-Bernard (2009) «Innovation culturelle, tourisme et gouvernance», chapitre 16 DANS Patrimoine culturel et tourisme alternatif: (Afrique, Amériques, Caraïbe, Europe), sous la direction de JeanMarie BRETON, p. 349-366. Paris : Éditions Karthala.

CAZES, Georges et Françoise POTIER (1998) Le tourisme et la ville : expériences européennes, Paris : L'Harmattan, coll. «Tourismes et Sociétés». $198 \mathrm{p}$.

CHAMEROY, Fabienne et Jean-Louis CHANDON (2010) «Les labels de qualité ont-ils des effets sur le comportement d'achat du consommateur?», Communication pour le $9^{e}$ Congrès des Tendances du Marketing en Europe (Venise : 21-23 janvier 2010), <http://www.marketingtrendscongress. com/2010_cp/Materiali/Paper/Fr/Chameroy_Chandon.pdf $>$, consulté en septembre 2010.

CHOAY, Françoise (1992) L'allégorie du patrimoine, Paris : Seuil. 271 p.

CLARKSON, Max B. (1995) «A stakeholder framework for analyzing and evaluating corporate social performance», Academy of Management Review, vol. 20, n 1, p. 92-117.

COCHRANE, Janet et Richard TAPPER (2006) «Tourism's Contribution to World Heritage Site Management», chapitre 7 DANS Managing World Heritage Sites, sous la direction de Anna LEASK et Alan FYALL, p. 97-109. London : Elsevier.

COURVOISIER, François H. et Cécile AGUILLAUME (2011) «L'inscription au Patrimoine mondial de l'UNESCO : un instrument de marketing territorial», <http://www.marketing-trends-congress.com/2011_cp/ Materiali/Paper/Territorial/Courvoisier_Aguillaume.pdf $>$, consulté en mars 2011. 
COUSIN, Saskia (2006a) "Le «tourisme culturel», un lieu commun ambivalent », Anthropologie et Sociétés, vol. 30, n 2, p. 153-173.

COUSIN Saskia (2006b) «De l'UNESCO aux villages de Touraine : les enjeux politiques, institutionnels et identitaires du tourisme culturel», Autrepart, $\mathrm{n}^{\circ}$ 40, p. 15-30.

DOTENWILL, Emmanuelle et Emmanuelle REYNAUD (2006) «Le rôle de l'approche par les parties prenantes dans l'initiation d'une politique de développement durable : étude du cas d'une entreprise de transport urbain ", Gestion 2000, nº 1, p. 33-59.

EL FAÏZ, Mohammed (2002) Marrakech, patrimoine en péril, Arles : Actes Sud Eddif. 188 p.

ERWANN, Charles et Hervé THOUÉMENT (2007) «Le label territorial, facteur d'attractivité touristique : une étude appliquée à la Bretagne», Téoros, p. 33-38. <http://teoros.revues.org/823>, consulté en mars 2011.

FIRAT, A. Fuat et Alladi VENKATESH (1995) «Liberatory Postmodernism and the Reenchantment of Consumption », Journal of Consumer Research, vol. 22, $\mathrm{n}^{\circ} 3$, p. 239-67.

FLAGESTAD, Arvid et Christine A. HOPE (2001) «Strategic success in winter sports destinations: a sustainable value creation perspective», Tourism Management, $\mathrm{n}^{\circ}$ 22, p. 445-461.

FREEMAN, Edward R. (1984) «Strategic Management: A Stakeholder Approach», Marshfield : Pitman Publishing. 276 p.

FREEMAN, Edward R. (1999) «Divergent Stakeholder Theory», Academy of Management Review, vol. 24, n 2, p. 233-236.

FREY, Bruno S. et Lasse STEINER (2010) «World Heritage List: Does it make sense? », Working Paper $n^{\circ} 484$, Zurich : University of Zurich, Institute for Empirical Research in Economics, p. 1-20, <www.iew.uzh.ch/wp/ iewwp484.pdf>, consulté en juillet 2010.

FRIEDMAN, Andrew L. et Samantha MILES (2006) Stakeholders: Theory and Practice, Oxford : Oxford University Press. 360 p.

GALLARZA, Martina G; Irene GIL et Garcia Haydée CALDERON (2002) «Destination Image: Towards a Conceptual Framework», Annals of Tourism Research, vol. 29, n 1, p. 56-78.

GIRAUD, Georges (2001) «Entre marques et labels : Comment s'orientent les choix des consommateurs? ", Revue Française du Marketing, n ${ }^{\circ} 183$ 184 , p. $169-179$.

GRAVARI-BARBAS, Maria et Sébastien JACQUOT (2008) «Impacts socioéconomiques de l'inscription d'un site sur la liste du Patrimoine Mondial : Une revue de la littérature», DANS Les impacts socio-économiques de l'inscription d'un site sur la liste du patrimoine mondial : trois études, sous la direction de Rémy PRUDHOMME, p. 17-74. Paris : Centre du patrimoine mondial de l'UNESCO.

GREFFE, Xavier (2003) La valorisation économique du patrimoine, Paris : La Documentation française. 383 p.

GUNN, Clare A. (2002) Tourism Planning: Basics, Concepts, Cases, $4^{\mathrm{e}}$ éd., Washington : Taylor \& Francis. 442 p.

HALL, Colin Michael et Stephen PAGE (2006) The Geography of Tourism \& Recreation. Environment, Place and Space, $3^{\mathrm{e}}$ éd., London : Routledge. 427 p.

HALL, Michael C. et Rachel PIGGIN (2003) «World Heritage Sites: Managing the Brand», chapitre 13 DANS Managing Visitor Attractions: New Directions, sous la direction de Alan FYALL, Brian GARROD et Anna LEASK, p. 203-219. Oxford : Butterworth-Heinemann.

HAMON, Viviane (2005) "Authenticité, tourisme durable et marketing", Revue Espaces, n 228 , p. 42-56.

HANKINSON, Graham (2010) «Place branding research: A cross-disciplinary agenda and the views of practitioners ", Place Branding and Public Diplomacy, vol. 6, n 4, p. 300-316.
ICOMOS - INTERNATIONAL CHARTER ON CULTURAL TOURISM, MANAGING TOURISM AT PLACES OF HERITAGE SIGNIFICANCE (1986) «Charte Internationale pour la sauvegarde des villes historiques», 6 décembre, <http://www.international.icomos.org/charters/towns_f. htm>, consulté en février 2010.

ICOMOS - INTERNATIONAL CHARTER ON CULTURAL TOURISM (1999) Managing tourism at places of heritage significance, <http:// www.international.icomos.org/charters/tourism_e.htm $>$, consulté en janvier 2009.

IVANOVIC, Milena (2009) Cultural Tourism, Cape Town : Juta Academic. $390 \mathrm{p}$.

KNAFOU, Rémy (2009) Les villes touristiques à patrimoine en Europe : Actes du colloque Tourisme urbain patrimoine et qualité urbaine en Europe (Rennes : 26 et 27 mars 2009), p. 6-11, <http://www.avecnet.net/publications/files/page71_4.pdf>, consulté en avril 2010.

KOTLER, Philip et David GERTNER (2002) «Country as brand, product and beyond: A place marketing and brand management perspective», Journal of Brand Management, vol. 9, $\mathrm{n}^{\circ}$ 4/5, p. 249-261.

LANDORF, Christine (2009) «Managing for sustainable tourism: a review of six cultural World Heritage Sites », Journal of Sustainable Tourism, vol. 17, $\mathrm{n}^{\mathrm{o}}$ 1, p. 53-70.

LARCENEUX, Fabrice (2003) «Segmentation des signes de qualité : labels expérientiels et labels techniques", Décisions Marketing, nº 29, p. 35-46.

LAZZAROTTI, Olivier (2000) «Patrimoine et Tourisme : un couple de la mondialisation», Mappemonde, $\mathrm{n}^{\circ}$ 57, p. 12-16.

LAZZAROTTI, Olivier (2010) «Tourisme culturel et patrimoine», Articulo revue de sciences humaines, <http://articulo.revues.org/1509>, consulté en octobre 2010.

LEASK, Anna et Alan FYALL (2006) Managing World Heritage Sites, Amsterdam : Elsevier. 289 p.

LORRAIN, Dominique (2000) «Gouverner les villes (questions pour un agenda de recherche)», Pôle Sud, no 13, p. 27-40.

MAJDOUB, Wided (2011) «Touristes des sites du patrimoine mondial : Vers quelles expériences? Une étude de la Médina de Sousse», DANS Patrimoine Mondial et Tourisme, sous la direction de Laurent BOURDEAU. Québec : Presses Universitaires du Québec (sous presse).

MARCOTTE, Pascale et Laurent BOURDEAU (2006) «Tourists Knowledge of the UNESCO Designation of World Heritage Sites: The Case of Visitors to Quebec City», International Journal of Arts Management, vol. $8, \mathrm{n}^{\circ} 2$, p. 4-13.

MARCOTTE, Pascale et Laurent BOURDEAU (2010) «La promotion des sites du Patrimoine mondial de l'UNESCO : Compatible avec le développement durable?», Management \& Avenir, vol. 4, nº 34, p. 270-288.

MCINTOSH, Alison J. et Richard C. PRENTICE (1999) «Affirming Authenticity: Consuming Cultural Heritage», Annals of Tourism Research, vol. $26, \mathrm{n}^{\circ} 3$, p. $589-612$.

MCKERCHER, Bob et Hilary DU CROS (2002) Cultural tourism: the partnership between tourism and cultural heritage, Binghamton : The Haworth Hospitality Press. 262 p.

MILLAR, Sue (2006) «Stakeholders and Community participation», chapitre 3 DANS Managing World Heritage Sites, sous la direction de Anna LEASK et Alan FYALL, p. 37-54. Amsterdam : Elsevier.

MINVIELLE, Jean-Paul; Mounir SMIDA et Wided MAJDOUB (2008) Tourisme Saharien et Développement Durable : Enjeux et Approches Comparatives : Actes du Colloque International de Tozeur (Tozeur : novembre 2007), 649 p., <http://horizon.documentation.ird.fr/exl-doc/ pleins_textes/divers09-09/010045751.pdf>, consulté en décembre 2009. 
MITCHELL, Ronald K.; Bradley R. AGLE et Donna J. WOOD (1997) «Toward a Theory of Stakeholder Identification and Salience: Defining the Principle of Who and What Really Counts ", The Academy of Management Review, vol. 22, nº 4, p. 853-886.

NOISETTE, Patrice et Franck VALLERUGO (1996) Le marketing des villes. Un défi pour le développement stratégique, Paris : les éditions d'Organisation. $423 \mathrm{p}$.

OMT - ORGANISATION MONDIALE DU TOURISME (2005) Tourisme et durabilité : un aperçu général, Département du développement durable du tourisme. 28 p. <http://www.ul.edu.lb/files/announcements/ annonce_21_148_77.pdf $>$, consulté en juillet 2010 .

ONTT - OFFICE NATIONAL DU TOURISME TUNISIEN (2009) Le tourisme tunisien en chiffres - 2009, Tunisie : Ministère du Tourisme, Office national du tourisme tunisien, Direction des Études. 61 p.

O'SHAUGHNESSY, John et Nicolas Jackson O'SHAUGHNESSY (2000) "Treating the nation as a brand: Some neglected issues", Journal of Macromarketing, vol. 20, $\mathrm{n}^{\circ}$ 1, p. 56-64.

OECD (2009) The Impact of Culture on Tourism, Paris : OECD, traduction française, p. 3-75. <http://www.oecd.org/dataoecd/35/5/42040218. pdf $>$, consulté en janvier 2010.

PEACOCK, Alan et Rizzo ILDE (2008) The Heritage Game. Economics, Politics, and Practice, Oxford : Oxford University Press. 209 p.

PICARD, Michel (2001) «Bali : vingt ans de recherches», Anthropologie et Sociétés, vol. 25, n² 2, p. 109-127.

PIKE, Steven (2005) "Tourism destination branding complexity», Journal of Product \& Brand Management, vol. 14, $\mathrm{n}^{\circ} 4$, p. 258-259.

PIKE, Steven D. (2009) «Destination brand positions of a competitive set of near-home destinations ", Tourism Management, vol. 30, $\mathrm{n}^{\circ} 6$, p. $857-866$

PRUD’HOMME, Rémy (2008) «Les impacts socio-économiques de l'inscription d'un site sur la liste du patrimoine mondial : trois études ", DANS Les impacts socio-économiques de l'inscription d'un site sur la liste du patrimoine mondial : trois études, p. 2-15. Paris : Centre du Patrimoine Mondial de l'UNESCO.

RICHARDS, Greg (1996) «European Cultural Tourism: Trends and Future Prospects", chapitre 17 DANS Cultural Tourism in Europe, p. 311-333. Oxon : CAB International.

RICHARDS, Greg (2001) «The experience industry and the creation of attractions », DANS Cultural Attractions and European Tourism, p. 55-69. New York : CABI Publishing.

RICHARDS, Greg (2002) «Tourism Attraction Systems Exploring Cultural Behaviour », Annals of Tourism Research, vol. 29, $\mathrm{n}^{\circ} 4$, p. 1048-1064.

RINGER, Greg (dir.) (1998) Destinations: Cultural landscapes of tourism, London : Routledge Advances in Tourism. 180 p.

RITCHIE, J. R. Brent et Geoffrey I. CROUCH (2000) «The Competitive Destination: a Sustainability Perspective», Tourism Management, vol. $21, \mathrm{n}^{\circ} 1$, p. 1-7.

ROBINSON, Mike et David PICARD (2006) Tourism, Culture and Sustainable Development, rapport UNESCO, Paris : UNESCO. 97 p.

ROSEMBERG, Muriel (1997) «Marketing urbain et projet de ville : parole et représentations géographiques des acteurs", Cybergeo : European Journal of Geography, <http://cybergeo.revues.org/1977>, consulté en novembre 2010.
ROSEMBERG, Muriel (2000) Le marketing urbain en question : production d'espace et de discours dans quatre projets de villes, Paris : Anthropos. $184 \mathrm{p}$.

RYAN, Jason et Sari SILVANTO (2009) «The World Heritage List: The making and management of a brand », Place Branding and Public Diplomacy, vol. 5 , n 4 , p. 290-300.

SAARINEN, Jarkko (2004) " "Destinations in change": The transformation process of tourist», Tourist Studies, vol. 4, $\mathrm{n}^{\circ}$ 2, p. 161-79.

SARANIEMI, Saila et Mika KYLÄNEN (2011) «Problematizing the Concept of Tourism Destination: An Analysis of Different Theoretical Approaches ", Journal of Travel Research, vol. 50, n 2, p. 133-143.

SIRAVO, Francesco (2009) Historic Cities and their Survival in a Globalized World: Proceedings of the tenth World Congress of the Organization of World Heritage Cities (Quito : 8-11 septembre), p. 14-35. <http://www. getty.edu/conservation/publications/pdf_publications/proceedings_ quito_eng.pdf>, consulté en mars 2010.

UNESCO (2002) Managing Tourism at World Heritage Sites: a Practical Manual for World Heritage Site Managers, rapport rédigé par Arthur PEDERSEN. Paris : UNESCO World Heritage Centre. 96 p.

UNESCO (2004) Patrimoine et développement durable dans les villes historiques du Maghreb contemporain : enjeux, diagnostics et recommandations, rapport final de la rencontre «Fès 2003 ", coordination scientifique D. Pini, Rabat : Bureau de l'UNESCO à Rabat. 257 p.

UNESCO (2006) Guide à l'attention des collectivités locales africaines : Patrimoine culturel \& développement local, s. 1. : édition CRATerreENSAG Convention France-UNESCO. 108 p.

VAN DER BORG, Jan (1998) «La gestion du tourisme dans les villes historiques", DANS Le tourisme et la ville : expériences européennes, sous la direction de Georges CAZES et Françoise POTIER, p. 99-110. Paris : L'Harmattan.

VAN DER YEUGHT, Corinne (2009) «Favoriser l'émergence d'un acteur stratégique dans les destinations touristiques pour répondre aux défis du développement durable», Management et Avenir, vol. 6, n 26, p. 300-317.

WTO - WORLD TOURISM ORGANIZATION (1985) The States' Role in Protecting and Promoting Culture as a Factor in Tourism Development and the Proper Use and Exploitation of the National Cultural Heritage of Sites and Monument for Tourists, Madrid : World Tourism Organization. $27 \mathrm{p}$.

WTTC - World Travel and Tourism Council (2010) Tourism: Progress \& Priorities 2009/2010, WTTC, 36 p. <http://www.wttc.org/>, consulté en septembre 2010.

YILMAZ, Burcu Selin et Ozgur Devrim GUNEL (2009) «The Importance of Strategic Stakeholder Management in Tourism Sector: Research on Probable Applications », Tourismos: an International Multidisciplinary Journal of Tourism, vol. 4, $\mathrm{n}^{\circ}$ 1, p. 97-108.

ZENKER, Sebastian et Erik BRAUN (2010) «The Place Brand Centre - A Conceptual Approach for the Brand Management of Places », Proceedings $39^{\text {th }}$ European Marketing Academy Conference (Copenhague, Danemark : 1-4 juin 2010), <http://www.placebrand.eu/mediapool/85/857874/data/Zenker_Braun_EMAC2010.pdf>, consulté en octobre 2010 . 